\title{
The role of physiotherapy in intensive care units: a critical review
}

\author{
DOI: https://doi.org/10.5114/pq.2019.87739
}

\author{
Narasimman Swaminathan ${ }^{1}$, Reshma Praveen ${ }^{2}$, Praveen Jayaprabha Surendran ${ }^{2}$ \\ ${ }^{1}$ Faculty of Physiotherapy, Sri Ramachandra Institute of Higher Education and Research, Chennai, India \\ 2 Hamad Medical Corporation, Doha, Qatar
}

\begin{abstract}
Physiotherapy has been accepted as an integral part of critical care in developed countries. Preventing and managing pulmonary complications of ventilated and other critically ill patients is still considered to be the primary task of physiotherapists in India. As movement experts of the intensive care unit team, physiotherapists play a major role in preventing neuromuscular complications through early mobilization and therapeutic exercise prescription in the developed countries. This critical review analyses the physiological rationale behind the physiotherapeutic technique and existing research evidence pertaining to the role of physiotherapy in critical care. The paper also highlights the simple evidence-based protocol which was successfully implemented in a tertiary care hospital of India.

Key words: critical care, physiotherapy, airway clearance, early mobilization
\end{abstract}

\section{Background}

Intensive care units (ICUs) play a vital role in any of the modern hospitals, with the aim to enhance the survival probability of people with acute illnesses or exacerbations of chronic illness. ICU requires a team of committed individuals to achieve this desired objective and the physiotherapist has established their role in this team. Physiotherapy has been acknowledged as an integral part of ICU management in most of the developed countries [1]. The rationale of physiotherapeutic interventions in any critical care unit is to avert pulmonary complications by removing airway secretions, as well as improving gaseous exchange and lung volumes [2]. Apart from pulmonary complications, critically ill patients are prone to develop other complications, such as muscular weakness and ICU-induced delirium.

Besides counteracting pulmonary complications, physiotherapy, as an integral part of critical care, prevents immobilization complications, assists in the weaning process from ventilatory dependence, and reduces the ICU stay. Physiotherapists employ various techniques, like positioning, postural drainage therapy, chest wall manipulations, manual hyperinflation $(\mathrm{MHI})$, early mobilization, and therapeutic exercises to assist in early recovery of critically ill patients [3]. A survey performed in Indian ICUs revealed that $79 \%$ of the centres had on-call physiotherapy services [4]. Subjects surviving critical illness suffer from increased fatigability, muscle weakness, and reduced mobility for a longer period after discharge. Early physiotherapy in the ICU is safe and feasible $[5,6]$.

Timely ICU physiotherapy reduces the span of ICU stay and duration of mechanical ventilation, as well as enhances the functional capacity. This critical review aims to analyse the physiological effects of various physiotherapy techniques and to discuss the existing evidence to substantiate the role of physiotherapy in ICU, especially from the point of view of developing countries.

\section{Body positioning}

Positioning is one of the most effective interventions used by physiotherapists. The concept of positioning, which is governed primarily by the influence of gravity, was based on the principles of the West lung model [7]. Thus, the principles of body positioning have a direct outcome in optimizing the respiratory mechanics and thereby enhancing gas exchange, oxygenation, and ventilation-perfusion matching in mechanically ventilated ICU patients $[8,9]$.

In the upright position, the superior portion of the pleural space is relatively more negative. Pleural pressure is more positive in the dependent basilar lung regions because of the downward acting weight of the lung. The lung is easier to inflate at low volumes than at high volumes, where it is compliant. Since the expanding pressure at the base of the lung is small and has a small resting volume, it expands well on inspiration. The apex of the lung has a large expanding pressure, a higher resting volume, and small change in volume during ventilation. The base of the lung has larger change in volume and smaller resting volume than the apex and hence its ventilation is greater. Therefore, the base of the lung is poorly expanded but better ventilated [7].

The different regional lung compliance is accountable for the dependent alveoli being relatively compliant and the non-dependent alveoli being relatively non-compliant. Thus, most of the tidal volume is preferentially distributed to the dependent alveoli because they expand more per unit pressure change than the non-dependent alveoli do. As a result, erect sitting may increase the lung compliance.

On the basis of these physiological benefits, therapeutic body positioning is used to augment arterial oxygenation by enhancing the gaseous exchange and reducing the work of breathing in subjects diagnosed with acute respiratory distress syndrome (ARDS) [10-12]. Lateral position is proved to be effective in mobilizing airway secretions and preventing

Correspondence address: Narasimman Swaminathan, Faculty of Physiotherapy, Sri Ramachandra Institute of Higher Education and Research, Porur, Chennai 600116, Chennai, Tamil Nadu, India, e-mail: narasimmansnathan@sriramachandra.edu.in

Received: 09.07.2019

Accepted: 16.08.2019

Citation: Swaminathan N, Praveen R, Surendran PJ. The role of physiotherapy in intensive care units: a critical review. Physiother Quart. 2019;27(4):1-5; doi: https://doi.org/10.5114/pq.2019.87739. 
atelectasis. Upright position is preferred to lower the work of breathing and to improve the lung volumes. When critically ill patients are turned for more than $40^{\circ}$, the incidence of nosocomial pneumonia becomes markedly reduced. The Centers for Disease Control and Prevention strongly recommends frequent positional changes [13].

In ventilated patients, therapeutic positioning is carried out with the intention to mobilize the secretion, prevent ventilator-associated pneumonia, improve the lung volumes and capacities, and facilitate weaning. The selection of therapeutic body positioning must be based on the day-to-day assessment findings and clinical reasoning. A survey in this regard revealed that most therapists opted for a position based on chest X-ray and auscultatory findings. Utmost care should be taken with the lines, tubes, and catheters while positioning. Various physiological parameters, like heart rate, fraction of inspired oxygen $\left(\mathrm{FiO}_{2}\right)$, positive end-expiratory pressure (PEEP), intracranial pressure, and mean arterial pressure, should also be considered [14].

\section{CLINICAL IMPLICATIONS}

Body positioning:

- Improves pulmonary function (gaseous exchange and breathing mechanics).

- Prevents ventilator-associated pneumonia.

- Assist in airway clearance.

- Is safe and requires sound clinical judgement by the health care providers.

\section{Airway clearance}

\section{Chest physiotherapy}

Patients admitted to critical care units, especially if mechanically ventilated, are at risk of retention of secretions. A range of techniques are employed by therapists to prevent the accumulation of secretions. Postural drainage therapy is still considered as a gold standard technique to mobilize the secretions in critically ill patients [15]. In this technique, gravityassisted positions are utilized to drain the secretions from the peripheral to the central airways. A total of 14 positions are used to drain the various lung segments.

Chest wall manipulations like percussion and vibration are usually carried out in gravity-assisted positions to aid secretion clearance. The mechanical energy produced by chest wall manipulations is transmitted to the airways and promotes the loosening and mobilization of secretions. It is believed that these techniques combined with $\mathrm{MHI}$ and endotracheal suctioning enhance airway clearance [16]. Mechanical chest wall percussion may be safe to use in secretion mobilization; it improves the ratio between partial pressure of oxygen in arterial blood $\left(\mathrm{PaO}_{2}\right)$ and $\mathrm{FiO}_{2}\left(\mathrm{PaO}_{2} / \mathrm{FiO}_{2}\right)$ and reduces the need for oxygen [17]. Chest wall squeezing during expiration enhances airway clearance during endotracheal suctioning [18].

Ntoumenopoulos et al. [19] proved that positioning and chest wall vibration reduced the occurrence of nosocomial pneumonia by $31 \%$ in mechanically ventilated patients in comparison with a control group receiving sham treatment. In a national survey [4], 91\% of Indian therapists working in ICUs reported that they performed postural drainage therapy in critically ill patients, which was in line with the responses by European participants (98\%). Some papers identified negative effects of percussion and vibrations.

\section{CLINICAL IMPLICATIONS}

- Postural drainage along with chest wall manipulations may be effective in clearing airway secretions in ventilated patients. This is not to be considered as a gold standard technique.

- Careful monitoring of vital signs during the procedures improves safety and efficacy.

\section{Manual hyperinflation and other airway clearance} techniques

During $\mathrm{MHI}$, the patient is discontinued from mechanical ventilation for a brief interval and high tidal volume is delivered through a manual resuscitation bag. The components of $\mathrm{MHI}$ include slow deep inspiration and inspiratory hold followed by quick release. The inspiratory hold is to recruit the alveoli through collateral ventilation. The quick release of the bag is to increase the expiratory flow [20,21].

In a randomized controlled trial, Patman et al. [22] analysed the effect of $\mathrm{MHI}$ on lung compliance, $\mathrm{PaO}_{2} / \mathrm{FiO}_{2}$ ratio, and the alveolar arterial oxygen gradient. They demonstrated a significant improvement in all the parameters when compared with the control group. Choi and Jones [23] observed an improvement in lung mechanics after $\mathrm{MHI}$ when compared with the control group. MHI was proved to be effective in reversing atelectasis in mechanically ventilated patients.

Four different types of equipment are used most commonly as per the literature to provide MHI in ICUs: Mapleson C, Magill, Laerdal, and Air Viva. The amount of pressure that is to be administered is not clear from literature, though the pressure used to inflate lungs in most studies was less than $40 \mathrm{~cm} \mathrm{H}_{2} \mathrm{O}$. Therefore, in view of complications, the pressure should be less than $40 \mathrm{~cm} \mathrm{H}_{2} \mathrm{O}$. Some authors suggested that the use of manometer in the circuit might prevent excess pressure. Utmost care has to be taken to ensure that PEEP does not exceed $10 \mathrm{~cm} \mathrm{H}_{2} \mathrm{O}[20,24]$.

Although there is limited evidence, $\mathrm{MHI}$ should not be used in patients with large emphysematous bullae or a bronchopleural fistula. Close monitoring is warranted while administering this technique since it may affect haemodynamic parameters. MHI application in Indian ICUs is not yet explored.

Other airway clearance techniques, like active cycle of breathing technique (ACBT) and positive expiratory pressure (PEP), are found to be effective in airway clearance in extubated patients. ACBT consists of cycles that involve breathing control (in which the patient is directed to breathe tidal volume), thoracic expansion, and forced expiratory technique. It was observed that ACBT prevented oxygen desaturation in patients with an acute exacerbation of chronic obstructive pulmonary disease. Our experience revealed that this was an effective technique immediately following extubation [25].

PEP is another airway clearance technique, in which the subject is asked to expire through resistance. The positive pressure produced during the expiration splints the airway from collapse and recruits airway collaterals. This mobilizes the secretions from the peripheral to the central airways.

\section{CLINICAL IMPLICATIONS}

- Manual hyperinflation seems to be an effective and safe method to clear airway secretions.. 


\section{Early mobilization}

Therapists working in ICUs utilize an assortment of techniques to avert or correct pulmonary complications and thus improve the functional status of the critically ill. The selection of the techniques is largely based on the assessment findings and clinical reasoning skills of the therapist. Amid all the techniques, early mobilization gained wide attention of critical care practitioners. This section of the article explores the importance of early mobilization in critically ill patients. Its first part discusses the effects of immobilization on various systems and the later half presents the effects of early mobilization and the safety issues to be considered while mobilizing critically ill patients.

\section{Effects of immobilization}

Critically ill ICU patients are immobilized owing to many reasons. It has been observed that the turning and repositioning happens only at an interval of more than 2 hours in critical care units and this may not be adequate to maintain the homeostasis. Bed rest is not benign. Immobilization affects virtually all the systems.

\section{Cardiovascular system}

Reversible myocardial dysfunction has been documented in critically ill patients. It is sometimes associated with systolic dysfunction, segmental contraction disturbances, and electrocardiographic changes. Some studies found decreased left ventricular size, stroke volume, cardiac output, and peripheral vascular resistance caused by prolonged bed rest. Physiologically, these changes may affect the coronary blood supply. The altered venous compliance due to immobilization will add to the risk of deep venous thrombosis and thromboembolism [26].

\section{Pulmonary system}

ICU immobilization reduces the strength of the respiratory muscles, which in turn affects the pulmonary function. Figure 1 explains the consequences of immobilization for pulmonary function. These problems will manifold if the patient is mechanically ventilated. Chest physiotherapy is being considered as an important adjunct in preventing pulmonary complications in critically ill patients.

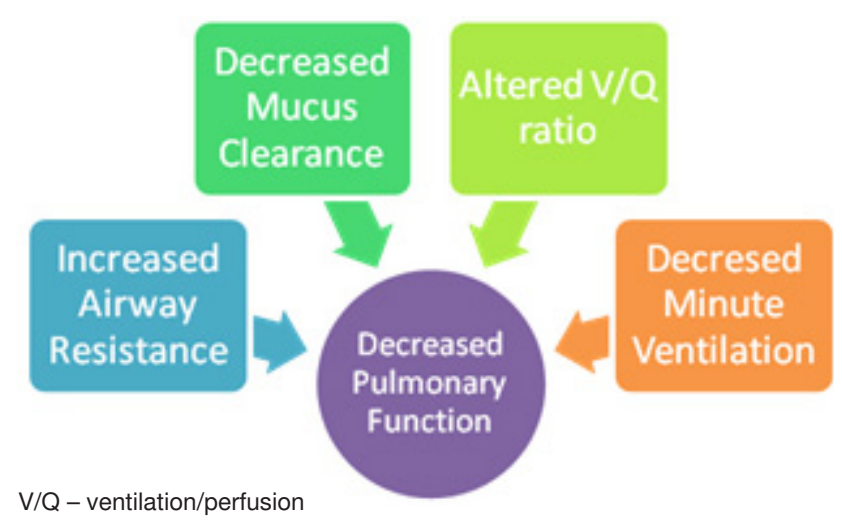

Figure 1. Consequences of immobilization for pulmonary function
Neurological system

It has been observed that immobilization in critical care units reduces the arousal level owing to altered cerebral electrical activities. Altered central nervous system activity will affect the breathing pattern, which may have an additional effect on the pulmonary function. Decreased postural reflexes and sympathetic stimulation is also documented in the literature [26].

\section{Neuromuscular system}

De Jonghe et al. [27] reported that up to $33 \%$ of mechanically ventilated patients experienced clinically evident neuromuscular weakness. After a week of immobilization, the skeletal muscle strength may decrease by up to $20 \%$, then further increasing with time. The pathophysiology of skeletal muscle weakness is multi-factorial in origin [28]. A therapist skilful in neuromuscular assessment will easily identify neuromuscular impairments caused by critical illness and immobilization.

Deconditioning effects on skeletal muscles include abnormal oxygen utilization during the activities of daily living. ICU-induced skeletal muscle weakness reduces the functional capacity and quality of life, which is well documented in the literature. In a study conducted by Herridge et al. [29], almost all ARDS survivors demonstrated decreased physical function, exercise tolerance, and quality of life.

Altered skeletal muscle functions affect the integrity of the joints, which further influences the functional activities of the individual. Decreased bone density is also reported in immobilized patients. Critical illness myopathy and neuropathy have been gaining attention recently $[30,31]$.

\section{Other systems}

The deteriorating effects of immobilization on other systems include: decreased cutaneous circulation and altered thermoregulation; decreased glomerular filtration, urinary output, and renal stasis; constipation resulting from decreased gut mobility; decreased insulin sensitivity and abnormal catecholamine metabolism.

\section{Role of physiotherapy in early mobilization}

The primary goal of physiotherapy as a part of ICU management is to prevent pulmonary complications and to initiate early mobilization. Traditionally, postural drainage, percussion, vibration, and simple forms of exercises are used by physiotherapists to achieve these goals. In the middle of 1970s, a walker with facilities to accommodate all the accessories, including mechanical ventilator, was proposed to make possible early mobilization of critically ill patients. It was reported that early ambulation of mechanically ventilated patients was safe and reduced the length of mechanical ventilation, thereby assisting in early weaning from the ventilator [32].

\section{Possible hindering factors in ICU mobilization}

Therapists working in ICUs are concerned about tubing and lines connected to the patients. In our experience, careful monitoring and precautionary measures prevent the dislodgement of tubing and other wires. It has been observed that therapists are apprehensive of acute haemodynamic changes due to mobilization. One should, however, keep in mind the positive effects of early mobilization. The multiplicity of vascular access sites is considered to be a potential barrier 
for mobilization in critically ill patients. Careful handling of the anatomical regions of vascular access by the therapists will prevent the detachment of the in-dwelling catheters. Prolonged use of sedation in critically ill patients may also affect the mobilization process. Other patient-related factors, like obesity, remain significant, too. Time constraint is an additional element perceived as a barrier for early mobilization [33].

\section{Practical considerations of early mobilization}

In routine clinical practice, early mobilization of critically ill patients is progressive in nature, as suggested by various authors [2, 3, 32, 34, 35]. We followed upright sitting, sitting at the edge of the bed, standing with support, spot marching, and supported walking as parts of early mobilization. Passive movements were not considered as early mobilization elements. The steps involved in our practice, designed on the basis of previous literature, are summarized in Table 1.

Table 1. Early mobilization protocol

\begin{tabular}{|c|l|}
\hline Phase & \multicolumn{1}{|c|}{ Steps } \\
\hline \multirow{2}{*}{1} & Sitting on the edge of the bed with or without support \\
\cline { 2 - 2 } & Standing with support \\
\cline { 2 - 2 } & Standing without support \\
\hline \multirow{2}{*}{2} & Transfer activities with support \\
\cline { 2 - 2 } & Initiation of walking with support \\
\hline \multirow{2}{*}{3} & Independent transfer activities \\
\cline { 2 - 2 } & Walking with support \\
\hline 4 & Progressive ambulation \\
\hline
\end{tabular}

The length of mobilization is increased progressively as per the patient's tolerance level. Mobilization frequency is also decided on the basis of the patient status; at least once daily is recommended to achieve the desired effects.

Precautions to be taken while mobilizing the patients are the following (Figure 2):

- Haemodynamic and oxygenation status should be evaluated before as well as throughout the course of mobilization. Ventilatory parameters should be noted.

- Drainage tubes, endotracheal/tracheostomy tubes should be secured. The mechanical ventilator and its tubing need utmost consideration.

- The arterial and venous catheters that can be disconnected temporarily and the lines that need to be present during mobilization should be identified.

- A portable monitor should be made available to observe the haemodynamic status during mobilization. Arm rest chair or a wheelchair should follow the patient during the mobilization to ensure patient safety and and assistance in the case of incompetence to complete the protocol.

- Oxygen supply should be ensured and the therapist should be well equipped for any emergency.

Mobilization should be withheld in the occurrence of the following conditions (Figure 2):

- Oxygen saturation $<88 \%$ during activities.

- Fall in blood pressure of more than $20 \mathrm{~mm} \mathrm{Hg}$ from baseline.

- Arrhythmias.

- Altered breathing pattern.

- Chest pain.

- Signs of intolerance.

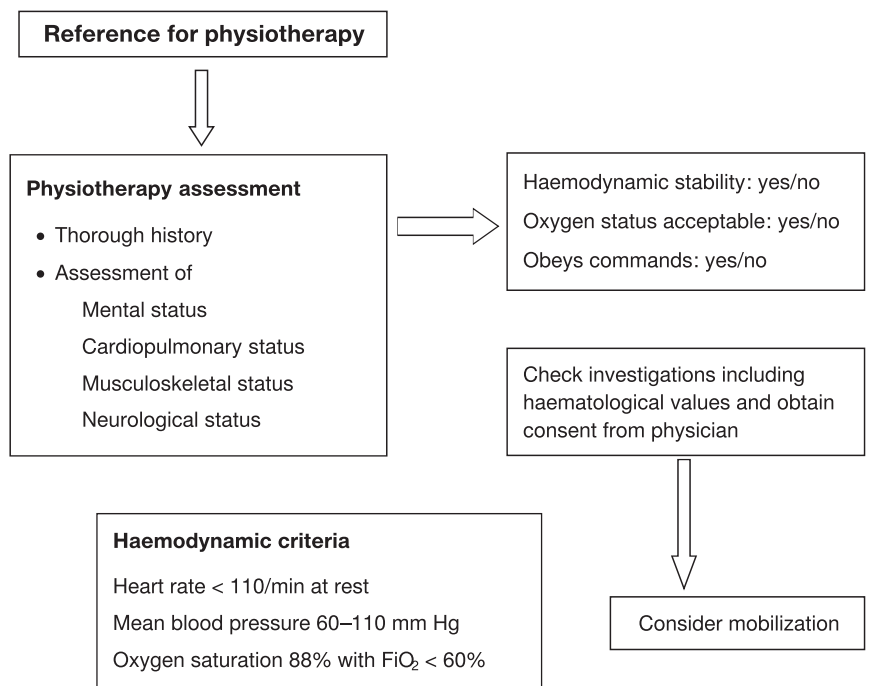

Figure 2. Early mobilization conditions

Early mobilization in critical care unit is proved to be effective in reducing the length of ICU stay and preventing complications. In our experience, we found it very useful and feasible to carry out. Well designed research in this regard is considered necessary to establish a viable protocol [35].

CLINICAL IMPLICATIONS

- Early mobilization is safe in ICUs.

- Clinical reasoning and decision making

by the therapist is the key.

- Consider phase-wise mobilization.

\section{Therapeutic exercises}

Prolonged ICU stay brings about muscle weakness and increased fatigability. Survivors of any critical illness require more support for a long period to resume efficient performance of their daily activities. Early rehabilitation of critically ill patients has proved to be effective in reducing the hospitalization time and dependency. Passive movements, active exercises, progressive mobilization, and other forms of activities utilizing a tilt table, treadmill, and bicycle are reported in the literature $[36,37]$.

In a prospective randomized control study, Nava [38] explored the effect of early pulmonary rehabilitation in patients with acute respiratory failure. A stepwise rehabilitation protocol was followed. The study demonstrated that early rehabilitation improved the functional autonomy when compared with the control group. Burtin et al. [39] investigated short-term effects of early exercises in critically ill patients. They revealed that additional exercise training by using a cycle ergometer for 20 minutes a day produced a significant improvement in the 6-minute walk distance and quadriceps strength. It also enhanced the subjects' feeling of well-being.

CLINICAL IMPLICATIONS

- Early rehabilitation is feasible and effective in ICUs.

- Progressive exercise prescription in ICUs reduces the hospital stay and may produce long-term effects.

\section{Conclusions}

Physiotherapy in the ICU or for critically ill patients plays a significant role. The choice of technique and protocol is individually tailored and multidimensional. But the effects of 
early physiotherapeutic rehabilitation are undoubtedly affirmative in ensuring speedy and maximal possible recovery of patients with critical illnesses. Well designed multicentre trials are warranted to establish physiotherapy effectiveness, especially from the perspective of developing countries.

\section{Ethical approval}

The conducted research is not related to either human or animal use.

\section{Disclosure statement}

No author has any financial interest or received any financial benefit from this research.

\section{Conflict of interest}

The authors state no conflict of interest.

\section{References}

1. Clini E, Ambrosino N. Early physiotherapy in the respiratory intensive care unit. Respir Med. 2005;99(9):10961104; doi: 10.1016/j.rmed.2005.02.024.

2. Stiller K. Physiotherapy in intensive care: towards an evidence-based practice. Chest. 2000;118(6):1801-1813; doi: 10.1378/chest.118.6.1801.

3. Stiller K. Physiotherapy in intensive care: an updated systematic review. Chest. 2013;144(3):825-847; doi: 10.1378/ chest.12-2930.

4. Kumar JA, Maiya AG, Pereira D. Role of physiotherapists in intensive care units of India: a multicenter survey. Indian J Crit Care Med. 2007;11(4):198-203; doi: 10.4103/ 0972-5229.37715.

5. Hodgson C, Denehy L, Ntoumenopoulos G, Santamaria J, Carroll S. An investigation of the early effects of manual lung hyperinflation in critically ill patients. Anaesth Intensive Care. 2000;28(3):255-261; doi: 10.1177/031 $0057 \times 0002800302$.

6. Hodgson CL, Stiller K, Needham DM, Tipping CJ, Harrold $\mathrm{M}$, Baldwin $\mathrm{CE}$, et al. Expert consensus and recommendations on safety criteria for active mobilization of mechanically ventilated critically ill adults. Crit Care. 2014;18(6):658; doi: 10.1186/s13054-014-0658-y.

7. West JB. Pulmonary gas flow and gas exchange. In: West JB (ed.), Respiratory physiology: people and ideas. New York: Oxford Press; 1966; 140-196.

8. Dean E, Ross J. Discordance between cardiopulmonary physiology and physical therapy: toward a rational basis for practice. Chest. 1992;101(6):1694-1698; doi: 10.1378/ chest.101.6.1694.

9. Dean E, Dean E. Oxygen transport: a physiologicallybased conceptual framework for the practice of cardiopulmonary physiotherapy. Physiotherapy. 1994;80(6): 347-354; doi: 10.1016/S0031-9406(10)61093-0.

10. RobakzPuthucheary Z, Harridge S, Hart N. Skeletal muscle dysfunction in critical care: wasting, weakness, and rehabilitation strategies. Crit Care Med. 2010;38(10 Suppl.):S676-S682; doi: 10.1097/CCM.0b013e3181f2 458d.

11. Herridge MS, Tansey CM, Matté A, Tomlinson G, DiazGranados N, Cooper A, et al. Functional disability 5 years after acute respiratory distress syndrome. N Engl J Med. 2011;364(14):1293-1304; doi: 10.1056/NEJMoa1011802.

12. Zink W, Kollmar R, Schwab S. Critical illness polyneuropathy and myopathy in the intensive care unit. Nat Rev Neurol. 2009;5(7):372-379; doi: 10.1038/nrneurol.2009.75.

13. Latronico N, Bolton CF. Critical illness polyneuropathy and myopathy: a major cause of muscle weakness and paralysis. Lancet Neurol. 2011;10(10):931-941; doi: 10.1016/S1474-4422(11)70178-8.

14. Truong AD, Fan E, Brower RG, Needham DM. Bench-tobedside review: mobilizing patients in the intensive care unit - from pathophysiology to clinical trials. Crit Care. 2009;13(4):216; doi: 10.1186/cc7885.

15. Morris PE. Moving our critically ill patients: mobility barriers and benefits. Crit Care Clin. 2007;23(1):1-20; doi: 10.1016/j.ccc.2006.11.003.

16. Perme C, Chandrashekar R. Early mobility and walking program for patients in intensive care units: creating a standard of care. Am J Crit Care. 2009;18(3):212-221; doi: 10.4037/ajcc2009598.

17. Da Conceição TMA, Gonzáles AI, de Figueiredo FCXS, Vieira DSR, Bündchen DC. Safety criteria to start early mobilization in intensive care units. Systematic review. Rev Bras Ter Intensiva. 2017;29(4):509-519; doi: 10.5935/0103-507X.20170076.

18. Berry MJ, Morris PE. Early exercise rehabilitation of muscle weakness in acute respiratory failure patients. Exerc Sport Sci Rev. 2013;41(4):208-215; doi: 10.1097/ JES.0b013e3182a4e67c.

19. Berney S, Haines K, Skinner EH, Denehy L. Safety and feasibility of an exercise prescription approach to rehabilitation across the continuum of care for survivors of critical illness. Phys Ther. 2012;92(12):1524-1535; doi: 10.2522/ptj.20110406.

20. Nava S. Rehabilitation of patients admitted to a respiratory intensive care unit. Arch Phys Med Rehabil. 1998; 79(7):849-854; doi: 10.1016/s0003-9993(98)90369-0.

21. Burtin C, Clerckx B, Robbeets C, Ferdinande P, Langer D, Troosters T, et al. Early exercise in critically ill patients enhances short-term functional recovery. Crit Care Med. 2009;37(9):2499-2505; doi: 10.1097/CCM.0b013e3181a 38937. 